\title{
The Continuum Slopes of Optically Selected QSOs
}

\author{
Paul J. Francis \\ School of Physics, University of Melbourne, Parkville \\ Victoria 3052, Australia. \\ E-mail: pfrancis@physics.unimelb.edu.au
}

Keywords: galaxies: quasars: general

\begin{abstract}
Quasi-simultaneous optical/near-IR photometry is presented for a sample of 37 luminous optically selected QSOs drawn from the Large Bright QSO Survey. Most of the QSOs have decreased in brightness since discovery; this is expected in flux-limited samples. The continuum shape of most of the QSOs can be represented by a power-law of the form $F(\nu) \propto \nu^{-0.3}$, but a few have softer (redder) continuum slopes.
\end{abstract}

\section{Introduction}

The shape of the continuum spectra of AGN in the rest-frame UV-optical region has long been uncertain. It is typically parameterised as a power-law of index $\alpha$, where the flux per unit frequency $f_{\nu} \propto \nu^{\alpha}$. The mean value of $\alpha$, the dispersion in values of $\alpha$, and even the validity of this power-law parameterisation, are all controversial.

For many years, a mean value of $\alpha=-0.7$ has been assumed by many researchers, based largely on the observations of Richstone \& Schmidt (1980) (see also O'Brien, Gondhalekar \& Wilson 1988 and Sargent, Steidel \& Boksenberg 1989). Recently, however, a number of authors have claimed that the true mean continuum slope is actually much harder (bluer) than this, with $\alpha=-0.3$ (eg. Neugebauer et al. 1987, Francis et al. 1991). There is equal disagreement about the range of continuum slopes shown by QSOs, with some authors claiming that all QSOs have essentially the same continuum slope (ie. little or no dispersion in $\alpha$, Sanders et al. 1989) while others claim that the dispersion in continuum slopes is large (Elvis et al. 1994, Webster et al. 1995).

These disagreements are important both physically, and because of the enormous uncertainty they introduce into measurements of QSO evolution. If, for example, the mean value of $\alpha$ is -0.3 , rather than -0.5 as commonly 
assumed, the number counts of bright QSOs at $z \sim 2$ can be explained with only half the evolution in the comoving density of luminous QSOs previously required, due to the difference in $k$-corrections. Allowing for a reasonable scatter in continuum slopes can also introduce factors of two or more into the inferred evolution of QSO number densities (Giallongo \& Vagnetti 1992, Francis 1993b).

Physically, a value of $\alpha \sim-0.3$ is consistent with the free-free emission models of Barvainis (1993), whereas a large dispersion in continuum slopes, unless caused by dust, would rule out this model. Webster et al. (1995) claim that flat-spectrum radio-loud quasars show a much greater dispersion in their continuum slopes, and a much softer (redder) mean slope than optically selected QSOs, and ascribe this difference to dust obscuration, a claim disputed by Serjeant \& Rawlings 1996, who ascribe the results to relativistically beamed red synchrotron emission.

The different values of $\alpha$ reported in the literature may reflect real differences between different classes of QSOs, such as radio-loud and radio-quiet, or luminous and faint. It is however possible that the differences reflect no more than different measurement techniques. One problem is that most AGNs with multi-wavelength observations are low luminosity, so the contribution of the host galaxy light is important (Elvis et al. 1994) and needs to be carefully corrected for. Measurement in the optical is complicated by the many strong blended Fe II emission-lines that obscure large parts of the spectra (Wills, Netzer \& Wills 1985, Francis et al. 1991). This obscuration makes measurements of continuum slopes inferred from optical data alone very unreliable, a problem that can be solved by the use of multi-waveband data. Alas, such data is seldom taken simultaneously, allowing variability to affect the inferred continuum slopes.

In this paper, an attempt is made to provide a definitive measurement of the continuum slope distribution of one type of quasars; luminous optically selected QSOs. The QSOs are drawn from the Large Bright QSO Survey (LBQS, Hewett, Foltz \& Chaffee 1995), a large, uniform and highly complete optically selected QSO sample that is increasingly being used as a standard against which to compare other quasar samples (eg. Webster et al. 1995). 
These QSOs are bright enough that the emission from their host galaxies can be neglected, as shown below. To minimise the effect of blended emission-lines, the continuum slopes are inferred from multi-waveband optical and near-IR photometry covering a factor of five in wavelength. The photometry is quasisimultaneous, to minimise any variability effects. This data set will provide a benchmark against which the continuum slopes of other samples can be compared.

The sample and observations are described in Section 2, the results are presented and discussed in Section 3, and conclusions drawn in Section 4.

\section{Observations}

2a. The Sample

A subset of QSOs drawn from the Large Bright QSO Survey (LBQS) were observed. The LBQS is described in Foltz et al. (1987, 1989), Hewett et al. (1991), Chaffee et al. (1991) and Morris et al. (1991), and summarised in Hewett, Foltz and Chaffee (1995). It consists of 1065 QSOs, with redshifts $0.2<z<3.4$, apparent magnitudes $16.0<B_{J}<18.85$, and absolute magnitudes $M_{B_{J}}<-21.5$ (for $q_{0}=0.5, H_{0}=100 \mathrm{~km} \mathrm{~s}^{-1} \mathrm{Mpc}^{-1}$ ). The QSOs were selected from UK Schmidt objective prism plates, using a variety of automated selection criteria, including continuum colour and the presence of strong emission-lines and continuum breaks. Comparison with existing surveys suggests that the LBQS is highly complete to its $B_{J}$ magnitude limit. Radio observations of a sub-set of the LBQS are presented by Visnovsky et al. (1992), Francis, Hooper \& Impey (1993) and Hooper et al. (1995). Discovery epoch $B_{J}$ magnitudes were obtained by scans of UK Schmidt plates and are accurate to $\sim 0.15$ mag.

$B, V, H$ and $K$ photometry for subset of 37 LBQS QSOs is presented in this paper. The subset observed were selected to be optically bright, high redshift, and accessible during the observing runs.

At redshifts $z \sim 2$, typical of most of the subsample observed, the $K$-band corresponds to the rest-frame $R$ band, and the $B$-band corresponds to restframe $1500 \AA$. We are therefore measuring the slope of the long-wavelength end of the 'Big Blue Bump' (Sanders et al. 1989). 
The spectral synthesis models of Bruzual \& Charlot (1993), and of RoccaVolmerange \& Guiderdoni (1988), were used to estimate the colours of the most luminous plausible host galaxies; starbursting giant proto-ellipticals. The rest-frame rest-frame $R$-band magnitudes at $z \sim 2$ were be calculated, and converted into observed $K$-band magnitudes. Even the most extreme galaxies had $K>17.5$; two magnitudes fainter than the QSOs in this sub-sample (Table 1). Host galaxy light is thus unlikely to contribute significantly to these observations.

\section{2b. Observations}

Optical photometry, in the $B$ and $V$ bands, was obtained on the Steward Observatory 90" telescope on the night of 3rd September 1992. Conditions were photometric, but due to malfunctions in both the autoguider and the tracking, fast manual guiding was required. Five minute CCD exposures were obtained. Not all objects could be imaged, due to substantial overheads associated with the manual guiding.

Near-IR photometry, in the $H$ and $K_{s}$ bands, was obtained on the nights of 27th and 28th April 1992, and 27th - 30th September 1992, on the Steward Observatory 61" telescope, using the $256 \times 256$ NICMOS HgCdTe array camera. $3 \times 3$ raster patterns were used, with three 50 second exposures at each grid pointing in $H$, and five 30 second exposures in $K_{s}$ to give an exposure time of 22.5 minutes. Conditions were photometric throughout.

The data was reduced using standard IRAF円 procedures. Magnitudes were measured using very large fixed apertures, as sky noise was not the dominant uncertainty, and the point spread function was very irregular due to the manual autoguiding.

Only the September data was used to derive continuum slopes; the optical and IR photometry are separated by three weeks, and so are not strictly simultaneous. Optically selected QSOs, however, show little variability of timescales of less than a month (Cristiani et al. 1996). Magnitude errors in both the optical and near-IR are $\sim 0.05$ magnitudes.

\footnotetext{
${ }^{1}$ IRAF is distributed by the National Optical Astronomy Observatories, which is operated by the Association of Universities for Research in Astronomy, Inc. (AURA) under cooperative agreement with the National Science Foundation.
} 


\section{Results and Discussion}

The results of the photometry are shown in Table 1 .

\section{3a. Variability}

The UK Schmidt sky survey plates from which the original magnitudes were measured were taken in the late 1970s and early 1980s. The time interval between the original $B_{J}$ magnitudes and our $B$ and $V$ photometry is thus $\sim 10$ years, allowing the assessment of the optical variability of the sample.

1992 epoch $B_{J}$ magnitudes were calculated using the approximate relationship $B_{J}=0.7 B+0.3 \mathrm{~V}$, and the change in $B_{J}$ between the Schmidt plate epoch and 1992 calculated. A histogram of the changes is shown in Figure 1.

As Figure 1 shows, the magnitude changes are very lopsided; of the 26 QSOs measured, 22 decreased in brightness over the $\sim 10$ years, while only 4 increased in brightness. The situation is even more lopsided when larger magnitude changes are considered; of the 16 QSOs which varied by more that 0.1 magnitudes, none increased in brightness. The median change in brightness is a decrease of 0.17 magnitudes, and the standard deviation is 0.23 magnitudes. This standard deviation is in good agreement with other studies (eg. Cristiani et al. 1996, and references therein). This lopsidedness remains regardless of the exact photometric techniques employed.

Why are the magnitude changes so lopsided? If QSO variation is a random walk process, the probability of a QSO increasing and decreasing in brightness will be equal. If this were true, the probability of getting such a lopsided distribution by chance is $0.6 \%$.

If, however, the variability of a QSO is not a random walk process, but each QSO has some characteristic brightness around which it varies, the lopsidedness can be explained. Any sample will consist of QSOs that were abnormally bright at the time of selection, and those that were abnormally faint. The QSO luminosity function is, however, very steep, so a QSO detected at a given magnitude is far more likely to be a fainter QSO caught in a bright state, than a brighter QSO caught in a faint state.

The size of the expected lopsidedness can be crudely estimated as follows. The typical variation is around $0.2 \mathrm{mag}$. Given the luminosity function of 
bright QSOs (eg. Boyle, Shanks and Peterson 1988), QSOs 0.2 magnitudes brighter than a given magnitude are $\sim \times 4$ less common than those 0.2 magnitudes fainter than the given magnitude. Thus the number of QSOs at the given magnitude which were at a maximum at the selection epoch is likely to exceed that which were at a minimum by a factor of $\sim 4$. Thus the ratio of the number QSOs that subsequently grow fainter to the number that grow brighter should be $\sim 4: 1$, in rough agreement to that observed.

\section{3b. Continuum Slopes}

Continuum slopes were computed as follows. Only QSOs with both optical and near-IR photometry were used. The optical magnitudes were corrected for the presence of strong broad emission-lines in the $B$ and $V$ bandpasses. For each spectrum, the redshift determined which strong emission-lines would be in each filter bandpass. If a strong line was present, its equivalent width in that QSO was obtained from the measurements of Francis (1993a). The flux in the filter bandpass was then corrected for the presence of this line, allowing for the redshift dependence of observed-frame equivalent widths. For the near-IR bandpasses, which often contained $\mathrm{H}-\alpha$ and $\mathrm{H}-\beta$, no measurements of the equivalent widths of individual QSOs were available, so the fluxes were corrected by assuming a mean rest-frame equivalent width of $58 \AA$ for $\mathrm{H}-\beta$ and $290 \AA$ for H- $\alpha$ (Francis et al. 1991).

For the higher redshift QSOs, Ly- $\alpha$ forest absorption can reduce the $B$ band flux. At $z>3$, the whole $B$-band is affected by Ly- $\alpha$ absorption, and no correction was attempted; the $B$-band flux was simply not used in the fit. For $2.4<z<3$ however, the $B$-band flux was corrected, the correction factor being chosen from visual inspection of the spectrum. Magnitude corrections (corrected magnitude minus observed magnitude) are shown in Table 2, and the corrected continuum fluxes in Table 3. Note that these corrections are typically small; even if no corrections are employed, the results of this paper are almost identical.

No attempt has been made to correct for weak emission-lines, and for Fe II emission. The use of a mean equivalent width for the Balmer line correction also introduces an error of $\sim 30 \%$ in the $H$ and $K$-band correction factors (Francis 1993a). However, the long wavelength baseline $(0.4-2.2 \mu \mathrm{m})$ means 
that these errors will not have a large effect on the derived continuum slope $\alpha$; a $25 \%$ error in one of the continuum fluxes introduces only an error of $\sim 0.1$ in the continuum slope index $\alpha$. Errors in $\alpha$ are predominantly systematic and hard to estimate, but from the self consistency of objects for which we have multiple observations, and the residuals to power-law fits to the continuum (Table 3), we estimate them to be of order 0.1.

A power-law continuum is fitted (by least-squares minimisation) to the corrected continuum fluxes; the resultant power-law indicies $\alpha$ are listed in Table 3. A histogram of these continuum slopes in shown in Figure 2. The power-law fit is generally a good one; the scatter around the fits (Table 3 ) is consistent with the photometry and correction errors. Note however that in the absence of $R$ or $I$-band photometry, we have little sensitivity to large-scale departures from a power-law continuum shape.

The mean value of the continuum slope index $\alpha$ is -0.46 , with a rootmean-squared (rms) dispersion of 0.30. Thus the mean slope is harder than the canonical $\alpha=-0.7$, but not as hard as the $\alpha=-0.3$ claimed by Francis (1993b). Note however that the median slope is -0.35 and the mode $\sim 0.3$; the mean being biassed towards higher values by the two outlying points. There are no obvious observational problems with the observations of these two outlying points; their redness is much too large to be explained by errors in the photometry and/or correction factors. Interestingly, both the outlying points are radio-loud QSOs, though the sample is too small to draw any significant conclusions.

These results demonstrate that the continuum slopes do show a real dispersion, but a much smaller one than that claimed by Webster et al. (1995) and Elvis et al. 1994). A standard deviation of 0.3 in $\alpha$ agrees with that found by Giallongo \& Vagnetti (1992), who showed that it leads to important consequences for measurements of QSO luminosity functions. The hard (blue) mean continuum slope reported here also suggests that previous workers, using $\alpha=-0.7$, will have overestimated the evolution of the number density of QSOs. A dispersion in $\alpha$ of 0.3 is smaller than that reported by Francis (1993b) for the LBQS as a whole (0.5); the difference is probably that the measurements here are more accurate, being based on a much wider wave- 
length coverage.

\section{Conclusions}

The very hard (blue) modal continuum slope of this sample agrees well with the measurements of Sanders et al. (1989), for another sample of luminous, optically selected QSOs. It is however, very different from the continuum slopes of the radio-selected sample of Webster et al. (1995) and the heterogeneous sample of Elvis et al. (1994), both of which show a substantial dispersion in continuum slopes, with $-0.3>\alpha>-4$. It is interesting to note, however, that the continuum slopes of the optically selected QSOs lie at the blue envelope of the continuum slope distribution of Webster et al.'s radio selected quasars.

Webster et al. suggested that the redness of their data was caused by dust obscuration, somewhere along the line of sight, and that the true continuum shape of QSOs is a uniform $f(\nu) \propto \nu^{-0.3}$. They showed that if this were true for radio-quiet QSOs, optically magnitude limited samples would show a distribution of continuum slopes with a narrow peak at $\alpha=-0.3$, and a low tail to redder slopes. This is a good description of our data (though given the small sample size, many equivalently good descriptions are possible).

Our observations are therefore consistent with a picture in which quasars have an intrinsic continuum slope of $\alpha \sim-0.3$ (as predicted by the free-free emission model), but are reddened by various amounts of dust, as suggested by Webster et al. Most of the heavily reddened quasars will be missed from samples, such as the LBQS, with a blue magnitude limit, but a few moderately red sources will remain, as observed. This model will be further elaborated in Francis et al. (1996, in preparation).

\section{Acknowlegements}

I wish to thank George and Marcia Rieke for their assistance in preparing for and reducing the IR observations, and Rachel Perkins for her help at the telescope. I acknowledge support from a NATO advanced fellowship, an ARC large grant, and National Science Foundation grant AST 90-01181. 


\section{References}

Barvainis, R. 1993, ApJ, 412, 513

Boyle, B. J., Shanks, T., \& Peterson, B. A. 1988, MNRAS, 235, 935

Bruzual, A. G., \& Charlot, S. 1993, ApJ, 405, 538

Chaffee, F. H., Foltz, C. B., Hewett, P. C., Francis, P. J., Weymann, R. J., Morris, S. L., Anderson, S. F., \& MacAlpine, G. M. 1991, AJ, 102, 461

Cristiani, S., Trentini, S., La Franca, F., Aretxaga, I., Andreani, P., Vio, R., \& Gemmo, A. 1996, A \& A, 30, 395

Elvis, M., Wilkes, B. J., McDowell, J. C., Green, R. F., Bechtold, J., Willner, S. P., Oey, M. S., Polomski, E., \& Cutri, R. 1994, ApJS, 95, 1

Foltz, C. B., Chaffee, F. H., Hewett, P. C., MacAlpine, G. M., Turnshek, D. A., Weymann, R. J., \& Anderson, S. F. 1987, AJ, 94, 1423

Foltz, C. B., Chaffee, F. H., Hewett, P. C., Weymann, R. J., Anderson, S. F., \& MacAlpine, G. M. 1989, AJ, 98, 1959

Francis, P. J. 1993a, ApJ, 405, 119

Francis, P. J. 1993b, ApJ, 407, 519

Francis, P. J., Hewett, P. C., Foltz, C. B., Chaffee, F. H., Weymann, R. J. \& Morris, S. L., 1991, ApJ, 373, 465

Francis, P. J., Hooper, E. J. \& Impey, C. D. 1993, AJ, 106, 417

Giallongo, E., \& Vagnetti, F. 1992, ApJ, 396, 411

Hewett, P. C., Foltz, C. B., \& Chaffee, F. H. 1995, AJ 109, 1498

Hewett, P. C., Foltz, C. B., Chaffee, F. H., Francis, P. J., Weymann, R. J., Morris, S. L., Anderson, S. F., \& MacAlpine, G. M. 1991, AJ, 101, 1121

Hooper, E. J., Impey, C. D., Foltz, C. B., \& Hewett, P. C. 1995, ApJ, 445, 62

Morris, S. L., Weymann, R. J., Anderson, S. F., Hewett, P. C., Foltz, C. B., Chaffee, F. H., Francis, P. J., \& MacAlpine, G. M. 1991, AJ, 102, 1627

Neugebauer, G., Green, R. F., Matthews, K., Schmidt, M., Soifer, B. T., \& Bennet, J. 1987, ApJS, 63, 615 
O'Brien, P. T., Gondhalekar, P. M., \& Wilson, R. 1988, MNRAS, 233, 801

Richstone, D. O., \& Schmidt, M. 1980, ApJ, 235, 361

Rocca-Volmerange, B., \& Guiderdoni, B. 1988, A \& AS, 75, 93

Sanders, D. B., Phinner, E.S., Neugebauer, G., Soifer, B. T., \& Matthews, K. 1989, ApJ, 347, 29

Sargent, W. L. W., Steidel, C. C., \& Boksenberg, A. 1989, ApJS, 69, 703

Serjeant, S., \& Rawlings, S. 1996, Nature, 379, 304

Visnovsky, K. L., Impey, C. D., Foltz, C. B., Hewett, P. C., Weymann, R. J., \& Morris, S. L. 1992, ApJ, 391, 560

Webster, R. L., Francis, P. J., Peterson, B. A., Drinkwater, M. J., \& Masci, F. J. 1995, Nature, 375, 469

Wills, B., Netzer, H., \& Wills, D. 1985, ApJ, 288, 94 
Table 1. Observed Magnitudes

\begin{tabular}{|c|c|c|c|c|c|c|c|}
\hline Name & $B$ & $\bar{V}$ & $\begin{array}{l}\text { Old } \\
B_{J}\end{array}$ & $H$ & $K_{s}$ & Redshift & $\begin{array}{l}\text { Radio } \\
\text { Loud? }\end{array}$ \\
\hline $0004+0147$ & 18.277 & 17.672 & 18.13 & 15.99 & 15.56 & 1.7110 & $?$ \\
\hline $0006+0200$ & 17.968 & 17.605 & 16.51 & 16.35 & 15.62 & 2.3358 & No \\
\hline $0006+0230$ & 18.569 & 18.339 & 17.99 & 16.83 & 16.00 & 2.0981 & No \\
\hline $0007+0142$ & 18.344 & 18.154 & 18.26 & 16.61 & 16.08 & 1.7654 & Yes \\
\hline $0009+0219$ & 18.360 & 17.960 & 17.99 & 15.91 & $\ldots$ & 2.6416 & No \\
\hline $0010-0012$ & 19.036 & 18.713 & 18.46 & .. & & 2.1536 & $?$ \\
\hline $0013-0029$ & 18.436 & 18.048 & 18.18 & & . & 2.0835 & No \\
\hline $0018-0220$ & 17.486 & 17.105 & 17.44 & 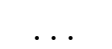 & 15.46 & 2.5960 & No \\
\hline $0049+0045$ & 17.766 & 17.540 & 17.50 & 15.74 & $\ldots$ & 2.2647 & No \\
\hline $0052-0058$ & 18.347 & 18.194 & 17.93 & 16.29 & 15.35 & 2.2120 & No \\
\hline 0056-0009 & 17.929 & 17.664 & 17.73 & 15.90 & $\ldots$ & 0.7175 & Yes \\
\hline $0106+0119$ & 18.498 & 18.171 & 18.34 & 15.12 & 14.04 & 2.0989 & Yes \\
\hline $0256-0000$ & 18.876 & 17.606 & 18.22 & 15.72 & 15.19 & 3.3638 & Yes \\
\hline $0256-0031$ & . & & 17.59 & 15.49 & 14.80 & 1.9951 & $?$ \\
\hline $0257-0254$ & $\ldots$ & $\ldots$ & 17.60 & 14.71 & 14.51 & 1.0700 & $?$ \\
\hline $0258+0210$ & 18.120 & 17.907 & 17.97 & 15.88 & 15.52 & 2.5239 & No \\
\hline $0301+0015$ & $\ldots$ & $\ldots$ & 18.31 & 16.44 & $\cdots$ & 1.6458 & $?$ \\
\hline $0302-0019$ & 18.409 & 17.436 & 17.78 & 15.87 & 15.27 & 3.2814 & No \\
\hline $1012+0213$ & $\ldots$ & $\ldots$ & 17.63 & 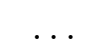 & 15.10 & 1.3778 & Yes \\
\hline $1013+0124$ & $\ldots$ & $\cdots$ & 16.52 & & 14.48 & 0.7790 & Yes \\
\hline $1215+1121$ & $\ldots$ & $\ldots$ & 16.61 & .. & 14.96 & 1.3984 & Yes \\
\hline $1216+1754$ & . & $\cdots$ & 18.07 & & 15.23 & 1.8097 & Yes \\
\hline $1222+1433$ & $\ldots$ & $\ldots$ & 17.11 & . & 14.85 & 1.3368 & No \\
\hline $1233+1524$ & $\cdots$ & $\ldots$ & 18.06 & $\ldots$ & 16.20 & 1.5441 & No \\
\hline $2230+0232$ & 18.387 & 18.038 & 18.05 & 16.43 & 15.72 & 2.1468 & No \\
\hline $2231-0015$ & 18.096 & 17.394 & 17.53 & 15.46 & 15.02 & 3.0150 & No \\
\hline $2231-0048$ & 17.623 & 17.241 & 17.57 & . & $\ldots$ & 1.2095 & $?$ \\
\hline $2231-0212$ & 18.357 & 18.065 & 18.15 & $\cdots$ & 15.80 & 1.9052 & $?$ \\
\hline $2239+0007$ & 18.796 & 18.453 & 18.28 & 16.32 & $\ldots$ & 1.4401 & $?$ \\
\hline $2241+0014$ & $\ldots$ & $\cdots$ & 17.57 & 17.72 & 15.53 & 2.1310 & No \\
\hline $2241+0016$ & 18.361 & 17.996 & 18.30 & 16.52 & 15.74 & 1.3936 & $?$ \\
\hline $2243+0141$ & 18.911 & 18.544 & 18.25 & 16.52 & $\ldots$ & 2.3140 & No \\
\hline $2244-0105$ & 18.091 & 17.680 & 17.95 & 15.91 & 15.29 & 2.0300 & No \\
\hline $2244-0208$ & 19.421 & 19.016 & 18.4 & 17.55 & $\ldots$ & 1.9682 & $?$ \\
\hline $2247+0135$ & 17.945 & 17.378 & 17.77 & $\cdots$ & $\cdots$ & 1.1284 & $?$ \\
\hline $2248+0127$ & 18.582 & 18.163 & 18.21 & $\cdots$ & 15.81 & 2.5585 & No \\
\hline $2350-0045 B$ & $\ldots$ & & 18.38 & & 15.69 & 0.4437 & $?$ \\
\hline
\end{tabular}


Table 2. Magnitude Correction Factors

\begin{tabular}{lcccc}
\hline Name & $B$ & $V$ & $H$ & $K$ \\
& Correction & to Observed Magnitude \\
\hline $0004+0147$ & 0.06 & 0.03 & 0.0 & 0.0 \\
$0006+0200$ & 0.10 & 0.10 & 0.08 & 0.28 \\
$0006+0230$ & 0.13 & 0.05 & 0.08 & 0.27 \\
$0007+0142$ & 0.14 & 0.03 & 0.0 & 0.0 \\
$0009+0219$ & 0.08 & 0.11 & 0.0 & $\ldots$ \\
$0018-0220$ & 0.12 & 0.07 & $\ldots$ & 0.0 \\
$0049+0045$ & 0.07 & 0.09 & 0.08 & $\ldots$ \\
$0052-0058$ & 0.30 & 0.0 & 0.08 & 0.27 \\
$0056-0009$ & 0.08 & 0.0 & 0.0 & $\ldots$ \\
$0106+0119$ & 0.19 & 0.0 & 0.08 & 0.27 \\
$0256-0000$ & $\ldots$ & 0.57 & 0.0 & 0.08 \\
$0258+0210$ & 0.04 & 0.13 & 0.09 & 0.0 \\
$0302-0019$ & $\ldots$ & 0.48 & 0.0 & 0.08 \\
$2230+0232$ & 0.13 & 0.0 & 0.08 & 0.27 \\
$2231-0015$ & $\ldots$ & 0.0 & 0.0 & 0.08 \\
$2231-0212$ & 0.06 & 0.04 & $\ldots$ & 0.08 \\
$2239+0007$ & 0.06 & 0.0 & 0.28 & $\ldots$ \\
$2241+0016$ & 0.06 & 0.0 & 0.28 & 0.0 \\
$2243+0141$ & 0.18 & 0.10 & 0.08 & $\ldots$ \\
$2244-0105$ & 0.04 & 0.06 & 0.0 & 0.26 \\
$2244-0208$ & 0.09 & 0.11 & 0.0 & $\ldots$ \\
$2248+0127$ & 0.20 & 0.12 & $\ldots$ & 0.0 \\
\hline
\end{tabular}


Table 3. Corrected continuum fluxes

\begin{tabular}{lcccccc}
\hline Name & $0.44 \mu \mathrm{m}$ & $\begin{array}{c}0.55 \mu \mathrm{m} \\
\left(\text { flux } \times 10^{-29} \mathrm{~W} \mathrm{~m}^{-2} \mathrm{~Hz}^{-1}\right.\end{array}$ & $\begin{array}{c}\text { Residuals } \\
[\mathrm{mJy}])\end{array}$ & $\begin{array}{c}\text { Slope } \\
\alpha\end{array}$ \\
\hline $0004+0147$ & 0.205 & 0.294 & 0.438 & 0.411 & 0.035 & -0.377 \\
$0006+0200$ & 0.263 & 0.292 & 0.292 & 0.301 & 0.009 & -0.057 \\
$0006+0230$ & 0.147 & 0.156 & 0.188 & 0.214 & 0.005 & -0.220 \\
$0007+0142$ & 0.180 & 0.189 & 0.248 & 0.255 & 0.035 & -0.230 \\
$0009+0219$ & 0.155 & 0.209 & 0.472 & $\ldots$ & 0.004 & -0.735 \\
$0018-0220$ & 0.382 & 0.476 & $\ldots$ & 0.451 & 0.041 & -0.005 \\
$0049+0045$ & 0.325 & 0.314 & 0.513 & $\ldots$ & 0.016 & -0.390 \\
$0052-0058$ & 0.154 & 0.187 & 0.309 & 0.389 & 0.011 & -0.556 \\
$0056-0009$ & 0.278 & 0.305 & 0.476 & $\ldots$ & 0.000 & -0.417 \\
$0106+0119$ & 0.165 & 0.191 & 0.907 & 1.298 & 0.012 & -1.368 \\
$0256-0000$ & $\ldots$ & 0.189 & 0.562 & 0.768 & 0.007 & -1.048 \\
$0258+0210$ & 0.223 & 0.215 & 0.446 & 0.426 & 0.030 & -0.459 \\
$0302-0019$ & $\ldots$ & 0.240 & 0.489 & 0.499 & 0.027 & -0.564 \\
$2230+0232$ & 0.174 & 0.215 & 0.272 & 0.277 & 0.012 & -0.263 \\
$2231-0015$ & $\ldots$ & 0.391 & 0.714 & 0.628 & 0.066 & -0.400 \\
$2231-0212$ & 0.190 & 0.204 & $\ldots$ & 0.329 & 0.001 & -0.354 \\
$2239+0007$ & 0.127 & 0.148 & 0.250 & $\ldots$ & 0.002 & -0.513 \\
$2241+0016$ & 0.190 & 0.223 & 0.208 & 0.348 & 0.044 & -0.286 \\
$2243+0141$ & 0.103 & 0.124 & 0.250 & $\ldots$ & 0.002 & -0.675 \\
$2244-0105$ & 0.249 & 0.284 & 0.472 & 0.415 & 0.036 & -0.371 \\
$2244-0208$ & 0.070 & 0.079 & 0.104 & $\ldots$ & 0.002 & -0.293 \\
$2248+0127$ & 0.126 & 0.173 & $\ldots$ & 0.326 & 0.007 & -0.521 \\
\hline
\end{tabular}




\section{Figure Captions:}

Figure 1-The distribution of magnitude changes of QSOs, between the old, UK Schmidt $B_{J}$ magnitudes and those presented in this paper.

Figure 2-The distribution of continuum slopes, as measured by the powerlaw index $\alpha$, where the continuum flux per unit wavelength $f(\nu)$ if fit by a function of the form $f(\nu) \propto \nu^{\alpha}$. 


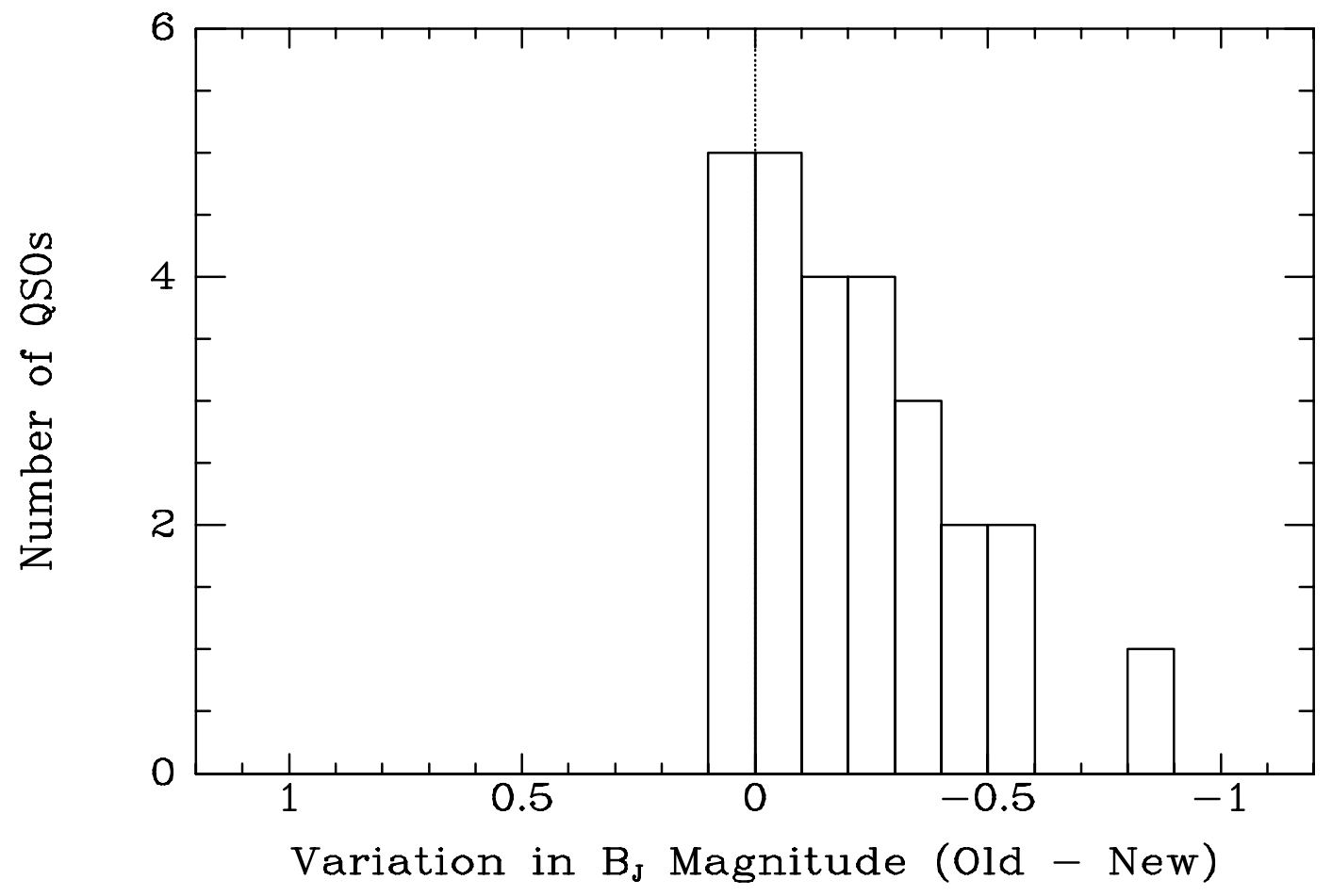




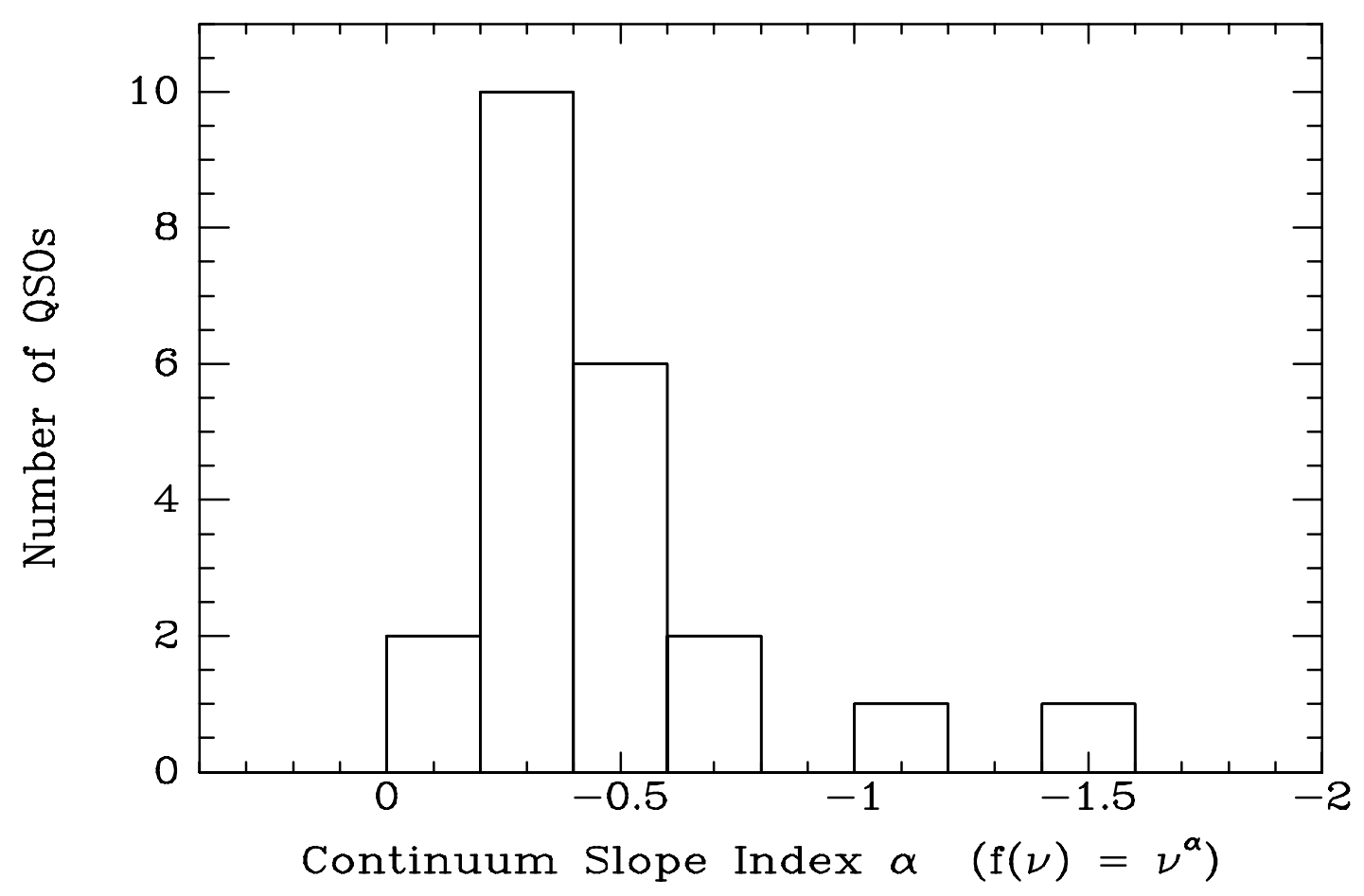

